\title{
Artificial Intelligence in Small Bowel Endoscopy: Current Perspectives and Future Directions
}

\author{
Dinesh Meher ${ }^{1}$ Mrinal Gogoi ${ }^{1} \quad$ Pankaj Bharali $\quad$ Prajna Anirvan $\quad$ Shivaram Prasad Singh ${ }^{1}$
} ${ }^{1}$ Department of Gastroenterology, S.C.B. Medical College, Cuttack,
Odisha, India

J Digest Endosc:2020;11:245-252

\begin{abstract}
Address for correspondence Shivaram Prasad Singh, MBBS, MD, DM, FSGEI, FACG, AGAF, FRCP [Edin], FRCPS [Glasgow], Department of Gastroenterology, S.C.B. Medical College, Cuttack, Odisha, 753007, India (e-mail: scb_gastro_dept@hotmail.com).
\end{abstract}

\begin{abstract}
Artificial intelligence ( $\mathrm{Al}$ ) is a computer system that is able to perform tasks which normally require human intelligence. The role of $\mathrm{Al}$ in the field of gastroenterology has been gradually evolving since its inception in the 1950s. Discovery of wireless capsule endoscopy (WCE) and balloon enteroscopy (BE) has revolutionized small gut imaging. While WCE is a relatively patient-friendly and noninvasive mode to examine the nonobstructed small gut, it is limited by a lengthy examination time and the need for expertise in reading images acquired by the capsule. Similarly, BE, despite having the advantage of therapeutic intervention, is costly, invasive, and requires general sedation. Incorporation of concepts like machine learning and deep learning has been used to handle large amounts of data and images in gastroenterology. Interestingly, in small gut imaging, the application of AI has been limited to WCE only. This review was planned to examine and summarize available published data on various Al-based approaches applied to small bowel disease.

Keywords We conducted an extensive literature search using Google search engine, Google - artificial intelligence

- machine learning

- deep learning

- wireless capsule endoscopy

- balloon enteroscopy

- artificial neural network

- convolutional neural network Scholar, and PubMed database for published literature in English on the application of different Al techniques in small bowel endoscopy, and have summarized the outcome and benefits of these applications of $\mathrm{Al}$ in small bowel endoscopy. Incorporation of $\mathrm{Al}$ in WCE has resulted in significant advancements in the detection of various lesions starting from dysplastic mucosa, inflammatory and nonmalignant lesions to the detection of bleeding with increasing accuracy and has shortened the lengthy review time in image analysis. As most of the studies to evaluate $\mathrm{Al}$ are retrospective, the presence of inherent selection bias cannot be excluded. Besides, the interpretability (black-box nature) of Al models remains a cause for concern. Finally, issues related to medical ethics and $\mathrm{Al}$ need to be judiciously addressed to enable its seamless use in future.
\end{abstract}

\section{Introduction}

Artificial intelligence (AI) involves computer programs that perform functions normally attributed to human intelligence, such as learning and problem solving. ${ }^{1} \mathrm{AI}$ has gradually evolved over the decades since its inception in the 1950s as a display of intelligent behavior indistinguishable from that of a human being and has come to

DOI https://doi.org/ $10.1055 / \mathrm{s}-0040-1717824$ ISSN 0976-5042. incorporate concepts like machine learning (ML) and deep learning (DL). DL, a relatively new concept, has emerged as a revelation in the realm of computer technology. A substantial amount of research has been done using DL application in the domain of image analysis in various medical fields including gastroenterology and hepatology. AI has been applied in the endoscopic analysis of nonmalignant

\footnotetext{
(C) 2020. Society of Gastrointestinal Endoscopy of India.

This is an open access article published by Thieme under the terms of the Creative Commons Attribution-NonDerivative-NonCommercial-License, permitting copying and reproduction so long as the original work is given appropriate credit. Contents may not be used for commercial purposes, or adapted, remixed, transformed or built upon. (https://creativecommons.org/licenses/by-nc-nd/4.0/).

Thieme Medical and Scientific Publishers Pvt. Ltd. A-12, 2nd Floor, Sector 2, Noida-201301 UP, India
} 
lesions such as polyps, ulcers, lymphangiectasia, angiectasia, etc. In addition to cancer detection and analysis of inflammatory lesions or localizing the lesion in obscure bleeding during wireless capsule endoscopy (WCE), AI has been used to good effect in diagnosis making and prognostication. The application of AI has waxed and waned over the past six decades with seemingly little improvement but various ML-based models like support vector machine (SVM), artificial neural network (ANN), and convolutional neural network (CNN) have proven to be useful in different branches of medicine with outstanding performance in image recognition and analysis. However, the optimal performance of DL-based methods requires a huge amount of properly labeled training data. This issue has been addressed by combining DL methods with reinforcement learning principles. ${ }^{2} \mathrm{~A}$ graphical summary of the concepts of $\mathrm{AI}, \mathrm{ML}$, and DL is depicted in - Fig. 1.

Nevertheless, AI and its newer models are not fool-proof or free from errors. The major drawbacks with newer techniques are overfitting and a lack of explainability. While the DL-based models perform much better than other models, these are intrinsically dependent on the training dataset. The lack of diversity and the presence of unidentified bias in the training dataset may hinder generalization to real-life situations and may lead to problems in proper model validation. Moreover, lack of explainability (blackbox nature) is a major concern in AI-based models.

Until now, most of the studies have stressed on improving the explainability of AI-based models. ${ }^{3}$ In the field of luminal gastroenterology, most reviews related to AI have focused on AI-assisted endoscopy, either in the form of automatic polyp detection in a colonoscopy ${ }^{4}$ or malignant lesion detection in the stomach and esophagus. ${ }^{5}$ In contrast, studies on AI-assisted endoscopy in diagnosing or localizing inflammatory, malignant, or bleeding lesions in the small bowel have been sparse. Studies on WCE have dealt only with the technical improvements of AI models in lesion detection, classification, and characterization. Surprisingly, data on the use of AI on single/double-balloon enteroscopy (DBE) are lacking. Furthermore, real-time

Artificial intelligence (AI) implemented by Machine learning (ML) and Deep learning (DL), DL is a newer subset of ML. AI conceptualized in 1950. ML and DL were discovered in 1980s, and 2010s, respectively.

\section{"AI" : Any technique which enables computers to mimic human behavior.} $1950 \mathrm{~s}$

"ML" : Ability to learn without being explicitly programmed. 1980 s

"DL" : A subset of machine learning which makesthe computation of multilayer neural network feasible. 2010 s

Fig. 1 Summary of the development of artificial intelligence, machine learning, and deep learning. applications of AI on any form of small bowel enteroscopy are lacking.

We conducted an extensive search in the internet using Google Scholar, Google search engine, and PubMed database on most recent and relevant articles using keywords: AI and small bowel endoscopy, computer-aided diagnosis (CAD), and capsule endoscopy, computer-based diagnosis in small bowel diseases, ML in small bowel endoscopies, and application of AI techniques in small bowel endoscopy. No exclusions were made on study designs and all articles were in English. In this present review, we have tried to summarize the different modalities of application of various AI models in diagnosing small bowel diseases with special emphasis on WCE and have dwelt on the pros and cons of such applications including their prospects.

\section{Small Bowel Endoscopy and AI Application}

WCE and balloon (single or double) enteroscopy have revolutionized the field of small gut imaging. It is no secret that the invention of WCE by Gavriel Iddan et $\mathrm{al}^{6}$ has brought in a sea change in the management of small-bowel diseases, including occult gastrointestinal (GI) bleeding, Crohn's disease, polyposis syndromes, malignancy, and celiac disease. Although its use is limited to the nonobstructed bowel, the advent of balloon enteroscopy (BE) to "chase" the findings of WCE has ushered in a new revolution in the realm of therapeutic interventions in the small bowel.

Advancements like suspected blood indicator, ${ }^{7}$ adaptive frame rate technology, ${ }^{8}$ and the quick-view algorithm ${ }^{9}$ based on AI for CAD have been developed to reduce the long review time and increase the accuracy in diagnosis. However, it is important to keep a note of the fact that these have yielded mixed results in terms of diagnosis and have reported high missing rates too. ${ }^{10}$

\section{Current Use of WCE in Practice}

Analysis of Malignant and Premalignant Lesions

As mentioned above, there have been only a few studies that have focused on small gut malignancies in particular. ${ }^{11-14}$ Technical variations on detection and characterization of polyps in WCE images have been tested in patients with suspected or previously known polyposis syndromes such as familial adenomatous polyposis and Peutz-Jeghers syndrome. ${ }^{15,16}$ WCE has also been found to be beneficial in the detection of polyps in the jejunum and ileum. ${ }^{15}$ WCE is found comparable to magnetic resonance enterography (MR enterography $)^{17}$ whereas computed tomography (CT) enterography and DBE have shown a superior sensitivity compared with WCE in the detection of small bowel tumors. ${ }^{18,19}$

Therefore, the presence of multiple constraints like lack of accuracy in image reading by an observer, lengthy capsule processing, and concerns regarding the image quality in WCE procedures coupled with the fact that the importance and applicability of AI have been steadily increasing led scientists to devise models that would be swift and would be less erroneous in detecting polyps and tumors in the small intestine. This, in turn, led researchers to focus primarily on domains 
like automatic polyp detection and characterization in the GI tract. Detection and characterization of a polyp are done using features (color, shape, edge, and texture) with various AI classifiers with a sensitivity of approximately $95 \%$ and accuracy varying from 91 to $98 \%$. The different methods of AI to detect and characterize polyp in WCE images/videos are summarized in - Table 1.

A few studies have also highlighted the methods of detecting polyp/malignant lesions in WCE images/videos concerning the small bowel using only various AI-based models, viz., ANN, KNN (K-nearest neighbors), multilayer perception network (MLP), and SVM ${ }^{11-14}$ with a reported sensitivity of 92 to $98 \%$ and a diagnostic accuracy of 92 to $97 \%$. The results of the AI studies involving small gut malignancies are summarized in - Table 1.

\section{Inflammatory and Other Nonmalignant Lesions}

Crohn's Disease

WCE is useful in the evaluation of Crohn's disease in the small intestine in cases where there is a diagnostic dilemma. Many studies have established the role of WCE as a valuable adjunct to conventional endoscopy and colonoscopy with ileoscopy with a reported sensitivity and specificity of 89 to $93 \%$ and 84 to $100 \%$, respectively. ${ }^{20,21}$ WCE has also been shown to be superior to CT enteroclysis ${ }^{22}$ and MR enterography, ${ }^{23}$ especially in terminal ileal disease and proximal small-bowel disease. ${ }^{22}$ Overall, most studies suggest a superior sensitivity of WCE with varying degrees of specificity over other radiological tests in the detection of small bowel Crohn's disease. It is, however, pertinent to remember that a lack of validated capsule criteria and the inability to obtain biopsy specimens for confirmation of diagnosis are significant limitations in the diagnosis of Crohn's disease. ${ }^{24}$ While DBE was found to be superior to WCE, ${ }^{25}$ false-positive results in a few asymptomatic patients raise concerns regarding accuracy in Crohn's disease. ${ }^{26}$ Further, severity scales for Crohn's disease using
WCE: the Lewis score and the capsule endoscopy Crohn's disease activity index have also been developed which are undergoing validation and these may prove useful in diagnosing Crohn's disease of the small bowel. ${ }^{27,28}$

To overcome the various limitations of WCE, researchers have tried to develop and modify AI-based models with considerable success. Various methods to determine features (color, edge, and texture), viz., mean shift algorithm ${ }^{29}$ and local binary pattern ${ }^{30}$ have been used to characterize inflammation in Crohn's disease. ML technique has also been used successfully in risk assessment of Crohn's disease and ulcerative colitis. Overall sensitivity and accuracy reported in the above studies are to the tune of 71 to $95 \%$ and 80 to $94 \%$, respectively. ${ }^{29-32}$ Studies showing the application of AI in patients of small gut IBD are summarized in - Table 2 .

\section{Celiac Disease}

Celiac disease (CD), with a worldwide incidence of $1 \%$, manifests as loss/scalloping of duodenal folds with nonspecific mucosal lesions (fissures, crevices, grooves, micro nodules, or a mosaic pattern) in the small intestine. ${ }^{33}$

Duodenal biopsies using standard endoscopy together with serological testing have been the cornerstone of diagnosis in $\mathrm{CD} .{ }^{34}$ The invasiveness of endoscopic biopsy and the expense of serological tests have resulted in the search for alternative economical, feasible, and noninvasive methods. Duodenoscopy, despite being convenient for inspecting and assessing villous atrophy in the duodenum, has significant limitations that it cannot examine the extent and severity of the disease. In this context, WCE may well be a suitable noninvasive, patient-friendly, and feasible alternative, which could visualize the entire small bowel for a detailed evaluation of the mucosal villous architecture with sufficient resolution in patients of suspected CD. ${ }^{35}$ Overall sensitivity and accuracy of 87 to $89 \%$ and $97 \%$, respectively are reported for the diagnosis of CD using WCE.

Table 1 Summaries of studies on polyp detection (overall) and tumor localization/characterization (in small bowel) involving Al

\begin{tabular}{|c|c|c|c|c|c|c|c|}
\hline Study & $\begin{array}{l}\text { Disease/ } \\
\text { localization }\end{array}$ & Design & $\begin{array}{l}\text { Feature/ } \\
\text { technique }\end{array}$ & Classifier & $\begin{array}{l}\text { Image/ } \\
\text { videos }\end{array}$ & Accuracy & Sensitivity \\
\hline $\begin{array}{l}\text { Nawarathna } \\
\text { et al }{ }^{14}\end{array}$ & Polyp(LB) & RS & Texton histogram & KNN, SVM & 400 & $95.27 \%$ & - \\
\hline Zhao et $\mathrm{al}^{20}$ & Polyp(LB) & RS & HMM & Boosted SVM & 1,200 & $90 \%$ & - \\
\hline Li et $\mathrm{al}^{21}$ & Polyp(LB) & RS & $\begin{array}{l}\text { Uniform LBP + } \\
\text { wavelet transform }\end{array}$ & SVM & 1,200 & $91.6 \%$ & - \\
\hline $\begin{array}{l}\text { Condessa } \\
\text { et a }{ }^{22}\end{array}$ & Polyp(LB) & RS & $\begin{array}{l}\text { Local polynomial } \\
\text { approximation }\end{array}$ & SVM & 3 videos & - & $92.31 \%$ \\
\hline $\begin{array}{l}\text { Constantinescu } \\
\text { et al }{ }^{11}\end{array}$ & Polyp (SB) & RS & WCE (SB) & ANN & $\begin{array}{l}54 \text { videos/90 } \\
\text { images }\end{array}$ & $97.7 \%$ & $93.8 \%$ \\
\hline Li et al ${ }^{12}$ & Tumor (SB) & RS & WCE (SB) & KNN, MLP & $\begin{array}{l}900 / 300 \\
\text { images }\end{array}$ & $90.5 \%$ & $92.3 \%$ \\
\hline Dinevari et al ${ }^{23}$ & Tumor (SB) & RS & WCE (SB) & SVM & $\begin{array}{l}600 / 200 \\
\text { images }\end{array}$ & $93.5 \%$ & $94.04 \%$ \\
\hline Liu et $\mathrm{a}^{13}$ & Tumor (SB) & RS & WCE (SB) & SVM & $\begin{array}{l}1,800 \\
\text { images }\end{array}$ & $97.3 \%$ & $97.8 \%$ \\
\hline
\end{tabular}

Abbreviations: Al, artificial intelligence; ANN, artificial neural network; CV, cross validation; HMM, hidden Markov model; KNN, k-nearest neighbors; LBP, local binary patterns; MLP, multilayer perception network; RS, retrospective study; SB, small bowel; SVM, support vector machine; WCE, wireless capsule endoscopy. 
Table 2 Summaries of studies on Crohn's disease and Celiac disease in small bowel involving Al

\begin{tabular}{|c|c|c|c|c|c|c|c|}
\hline Study & $\begin{array}{l}\text { Disease/ } \\
\text { localization }\end{array}$ & Design & $\begin{array}{l}\text { Diagnostic } \\
\text { modality }\end{array}$ & Al classifier & $\begin{array}{l}\text { Videos/ } \\
\text { Images in } \\
\text { training/ } \\
\text { testing }\end{array}$ & Accuracy & $\begin{array}{l}\text { Sensitivity/ } \\
\text { specificity }\end{array}$ \\
\hline Girgis et $\mathrm{al}^{29}$ & Crohn's disease & RS & WCE (SB) & SVM & $467 / 277$ images & 87 & $80 / 93$ \\
\hline Kumar et al ${ }^{30}$ & Crohn's disease & RS & WCE (SB) & SVM & 533 images & 80.2 & $81.1 / 93.6$ \\
\hline Wei et $a^{31}$ & Crohn's disease & RS & Genetics & SVM & $53,279 / 22,442$ & AUROC-0.86 & $71 / 83$ \\
\hline $\begin{array}{l}\text { Charisis and } \\
\text { Hadjileontiadis }^{32}\end{array}$ & Crohn's disease & RS & WCE (SB) & SVM & $800 / 102$ images & 93.8 & $95.2 / 92.4$ \\
\hline Ciaccio et al ${ }^{35}$ & Celiac disease & RS & WCE(SB) & SVM & $\begin{array}{l}8,600 / 10,000 \\
\text { images }\end{array}$ & 76.7 & $88 / 80$ \\
\hline Tenório et al $\left.\right|^{36}$ & Celiac disease & RS & EMR & $\mathrm{BI}, \mathrm{KNN}$ & 178/38 images & 80 & $78.8 / 80$ \\
\hline Gadermayr et al ${ }^{37}$ & Celiac disease & RS & $\mathrm{WL} / \mathrm{NBI}$ & SVM & 2,835 images & 99.6 & NA \\
\hline Zhou et a ${ }^{38}$ & Celiac disease & RS & EMR & GoogLeNet & $\begin{array}{l}8,800 / 8000 \\
\text { images }\end{array}$ & NA & $100 / 100$ \\
\hline Chen and Lee ${ }^{41}$ & Ulcer & RS & WCE(SB) & SVM & 250/930 images & 96.3 & $91.7 / 99.4$ \\
\hline Charisis et $\mathrm{al}^{40}$ & Ulcer & RS & WCE(SB) & SVM, MNN & $156 / 18$ images & 95 & $96.6 / 93.5$ \\
\hline Eid et $\mathrm{al}^{42}$ & Ulcer & RS & WCE(SB) & SVM & 260 images & 86.5 & $84.5 / 88.6$ \\
\hline Yuan et al ${ }^{43}$ & Ulcer & RS & WCE(SB) & SVM & $271 / 68$ images & 92.7 & $94.1 / 91.2$ \\
\hline
\end{tabular}

Abbreviations: Al, artificial intelligence; ANN, artificial neural network; BI, Bayesian inference; CD, Celiac disease; CV, cross-validation; EMR, electronic medical records; IBD, inflammatory bowel disease; KNN, K nearest neighbor; MNN, Multilayer neural network; NBI, narrow band imaging; RS, retrospective study; SB, small bowel; SVM, support vector machine; SVM, support vector machines; WCE, wireless capsule endoscopy; WL, white light.

Over the last decade, AI has been evolving in diagnosing and classifying disease severity in CD using WCE. The features (color, texture, and shape) of a lesion are being utilized for diagnosis and assessment of extent and severity of CD. ${ }^{35}$ A web-based clinical decision support system that uses AI techniques to diagnose $\mathrm{CD}$ cases has also been reported. ${ }^{36} \mathrm{~A}$ few researchers assessed a hybrid approach that incorporated expertise and technical knowledge into the computer-based classification, which showed a very high accuracy in diagnosing $\mathrm{CD}^{37} \mathrm{~A}$ 22-layered deep CNN named GoogLeNet achieved $100 \%$ sensitivity and specificity in diagnosing CD from WCE clips. $^{38}$ However, another group of researchers who built a series of predictive models to diagnose $\mathrm{CD}$ utilizing a variety of statistical approaches met with unsatisfactory results, yielding poor discriminatory performance with AUCs ranging from 0.49 to 0.53 . Overall sensitivity and accuracy reported in the above studies are 78 to $100 \%$ and 76 to $99 \%$, respectively. ${ }^{35-38}$ - Table 3 summarizes the results of studies on CD using AI.

\section{Ulcer}

A vast majority of WCE-related literature is concerned with the reduction of the examination time of WCE data in the detection of certain disorders in the small gut. However, there have been very few studies that have dealt with the detection of ulcers (7\%) and Crohn's lesions (2\%). ${ }^{39}$ Detection of such lesions is difficult owing to the inherent challenges like nonspecific characteristics of such lesions and the huge diversity in appearance. Time, expertise, and feasibility also remain a matter of concern for definitive identification and localization. Various methods have been utilized to extract the color and texture of ulcers while a few studies have focused on the salient region identification. Color-texture extraction using segmentation scheme, ${ }^{40}$ ulcer salient map redefined with Gabor filter, ${ }^{41}$ texture only extraction method based on discrete curvelet transform, ${ }^{42}$ and saliency map using super-pixel region ${ }^{43}$ are some of the techniques that have been successfully used. These methods have been reported to achieve a sensitivity of 84 to $97 \%$ while the overall diagnostic accuracy remains 86 to $96 \%$. The results of the AI-based studies on ulcers are summarized in - Table 2.

\section{Other Non-Inflammatory and Nonmalignant Lesions}

\section{Lymphangiectasia}

Lymphangiectasia is the pathologic dilation of lymphatic channels. When it occurs in the intestines, it is known as intestinal lymphangiectasia, also simply called lymphangiectasis. WCE is of use in the detection of these lesions in patients presenting with features of protein-losing enteropathy or chronic malnutrition. ${ }^{44}$ Here again, the variability in color, shape, and textural characteristics of lymphangiectatic lesions often makes it extremely difficult to characterize the lesion using WCE or BE, thus making the role of AI even more important. ${ }^{45}$ Algorithms using luminance information in hue, saturation, and intensity colors space and Commission Internationale de l'éclairage-Laboratory representation have reported a sensitivity and accuracy of approximately 48 and approximately $98 \%$, respectively in the detection of lymphangiectatic lesions in the small bowel.

\section{Hookworm Infestations}

Intestinal hookworms are difficult to find with direct visualization because of their small tubular structures and semitransparent features, which make it tough to distinguish them from the intestinal mucosa. Moreover, the presence 
Table 3 Summaries of studies on identification of non-inflammatory lesions and obscure gastrointestinal bleed in small bowel involving $\mathrm{Al}$

\begin{tabular}{|c|c|c|c|c|c|c|c|}
\hline Study & $\begin{array}{l}\text { Disease/ } \\
\text { localization }\end{array}$ & Design & $\begin{array}{l}\text { Diagnostic } \\
\text { modality }\end{array}$ & Al Classifier & $\begin{array}{l}\text { Videos/Images in } \\
\text { training/testing }\end{array}$ & Accuracy & $\begin{array}{l}\text { Sensitivity/ } \\
\text { specificity }\end{array}$ \\
\hline Cui et $\mathrm{al}^{45}$ & Lymphangiectasia & RS & WCE(SB) & Threshold SVM & 7,218 images & 97.9 & 48.8/NA \\
\hline He et $a^{47}$ & Hookworm & RS & WCE(SB) & CNN & $20,000-30,000$ & 88.5 & $84.6 / 88.6$ \\
\hline Wu et $a^{48}$ & Hookworm & RS & WCE(SB) & Rusboost SVM & $\begin{array}{l}401,476 / 40,148 \\
\text { images }\end{array}$ & 78.2 & $77.2 / 77.9$ \\
\hline Li and Meng ${ }^{56}$ & Obscure GI bleed & RS & WCE(SB) & MLP & $2,700 / 900$ & NA & $87.8 / 88.6$ \\
\hline Pan et $a^{57}$ & Obscure GI bleed & RS & WCE(SB) & PNN & $\begin{array}{l}14,630 \\
\text { images/150 videos }\end{array}$ & 87.4 & $93.1 / 85.8$ \\
\hline Lv et $\mathrm{al}^{58}$ & Obscure GI bleed & RS & WCE(SB) & SVM & $280 / 280$ & 97.9 & $97.8 / 98$ \\
\hline Fu et al ${ }^{59}$ & Obscure GI bleed & RS & WCE(SB) & SVM & $30,000 / 30,000$ pixels & 94 & $97 / 92$ \\
\hline Sainju et $a^{60}$ & Obscure GI bleed & RS & WCE(SB) & MLP & 100 images & 93 & $96 / 90$ \\
\hline $\begin{array}{l}\text { Hassan and } \\
\text { Haque }^{61}\end{array}$ & Obscure GI Bleed & RS & WCE(SB) & SVM & $1,200 / 1,720$ images & 99.2 & $99.4 / 99$ \\
\hline $\begin{array}{l}\text { Leenhardt } \\
\text { et } \mathrm{al}^{63}\end{array}$ & Obscure GI bleed & RS & WCE(SB) & CNN & $600 / 600$ images & 98 & $100 / 96$ \\
\hline Tsuboi et al ${ }^{64}$ & Obscure GI bleed & RS & WCE(SB) & CNN & $2,237 / 10488$ images & NA & $98.8 / 98.4$ \\
\hline
\end{tabular}

Abbreviations: Al, artificial intelligence; CNN, convolutional neural network; $\mathrm{Gl}$, gastrointestinal; MLP, multilayer perceptron network; MNN, multilayer neural network; RS, retrospective study; SB, small bowel; SSMD, single shot multibox detector; SVM, support vector machines; WCE, wireless capsule endoscopy.

of intestinal secretions makes detection even more difficult. The role of WCE in detecting hookworms in the small intestine has been mentioned in the literature, albeit with highly variable detection rates. ${ }^{46}$ Interestingly, AI methods have been utilized to detect hookworms in the small bowel with a sensitivity of 77 to $84 \%$ and accuracy of 78 to $88 \%$. The flip side of this is the reportedly high missing rates of around $23 \%{ }^{47,48}$ - Table 3 summarizes the results of the studies on noninflammatory lesions of the small gut using AI-based methods.

\section{Obscure GI Bleed}

Obscure gastrointestinal bleeding (OGIB) is defined as the bleeding from the digestive tract, which recurs or persists after a negative initial evaluation, using both upper and lower GI endoscopy and a negative result on radiologic imaging using small bowel follow-through or enteroclysis. ${ }^{49}$ Accounting for approximately $5 \%$ of overall GI bleeding, OGIB bleed has been shown to arise from the small bowel distal to the Ampulla of Vater and proximal to the ileocecal valve in more than $80 \%$ of the cases, rendering it relatively inaccessible to traditional endoscopy. ${ }^{49}$ While the efficacy and reliability of WCE have been tested over many years, the detection rate is variable ranging from 35 to $77 \%$. 50,51

Various studies comparing WCE to other methods in unraveling the causes of OGIB have shown it to be superior to the other investigations. WCE performed better than CT angiography, ${ }^{52}$ CT-enteroclysis, intraoperative enteroscopy, ${ }^{53}$ and push enteroscopy ${ }^{54}$ in the detection of the lesion. However, the detection rate is comparable to DBE. ${ }^{55}$

Despite the obvious advantages of WCEs over the traditional techniques, issues like exhaustive evaluation of images and interobserver/intraobserver variability including subjective humane error have plagued this novel technique. In a bid to overcome these problems, the application of different AI methodologies has been tried with variable success rates. Combining color and spatial information of bleeding lesions, viz., chromaticity moment ([HIS Color space $],^{56}[\text { RGB Color space }]^{57}$ ) and color descriptors (pyramid of color invariant histograms, pyramid of hue histograms, and pyramid of transformed color histograms), ${ }^{58}$ researchers have applied AI models to detect and localize bleeding lesions in the small bowel. Besides, a few studies have also explored the use of textural features such as pixel segmentation and pixel grouping ${ }^{59}$ while a few others have utilized statistical features like a color histogram. ${ }^{60} \mathrm{~A}$ novel algorithm that operates on normalized gray level co-occurrence matrix using the frequency spectrum of WCE images has also successfully been used. ${ }^{61}$ Further, AI methods like CNN, MLP, SVM, and PNN have also been used to classify the above image patterns for lesion detection and localization with a sensitivity of 87 to $100 \%$, specificity of 85 to $99 \%$, and a diagnostic accuracy ranging from 87 to $99 \%$.

Small-bowel angiectasia comprises the majority of small-bowel vascular lesions and is diagnosed in 30 to $40 \%$ of OGIB cases. ${ }^{62}$ Although detection of angiectasia using WCE is well established, computer-aided detection methods have not been validated as yet. CNN algorithm using still frames featuring annotated angiectasia ${ }^{63}$ and $\mathrm{CNN}$ algorithm based on single shot multibox detector using WCE images of angiectasia ${ }^{64}$ have been applied with a reported sensitivity of 98 to $100 \%$, specificity of 96 to $98 \%$, and accuracy of $98 \%$. - Table 3 summarizes the results of AI-based studies on small gut for the evaluation of OGIB.

\section{Challenges in $\mathrm{Al}$ and Future Directions}

Soon, AI is envisaged to play a major role in helping establish diagnoses, devising treatment protocols, and in the prediction of treatment outcomes. Over the last seven decades, AI has been 
studied extensively and important developments have been made which show promising results. However, a major drawback of all these endeavors is the retrospective nature of most of these studies which have heavily banked upon data chosen from specific endoscopic modalities limited to a fewer number of institutions. In such a situation, there is a high likelihood of selection bias creeping in, therefore, it is crucial to validate the performance of AI using different population-based models in a "real world" setting. Overfitting and spectrum bias have also been observed to impact negatively on Al performance in terms of reproducibility. Overfitting occurs when the learning model is dependent too much on a training dataset, resulting in unsuitable generalization to newer dataset leading to overfitting. ${ }^{65}$ There have indeed been attempts to find a way out to solve this problem but with limited success. Besides, datasets in case-control design studies are readily vulnerable to spectrum bias. Spectrum bias occurs when the training dataset does not adequately represent the target population. ${ }^{66}$ Because overfitting and spectrum bias may overestimate the accuracy of a model, external validation of unused datasets is mandatory. Additionally, robust clinical verification, as well as properly designed multicenter prospective studies with adequate criteria (inclusion/exclusion) representing the target population, is required. Furthermore, a lack of interpretability or explainability (black box nature) is a major concern in AI technology where the decision-making mechanism of AI models may not be clearly understood and corrected if needed. Some techniques have been developed to address "black box" limitations such as the attention mapping and saliency region identification; these, however, require further studies regarding their applicability. ${ }^{67}$ As the accuracy and efficiency of ML model is proportional to the input data, developing an efficient ML model is challenging due to the paucity of human-labeled data. Data augmentation strategies have been proposed to address this problem. ${ }^{68}$ Of note Spiking neural networks, which closely mimic the biological mechanisms of neurons, can potentially replace the present ANN models, bringing in higher and more sophisticated computational ability. ${ }^{69}$

The diagnostic precision of AI does not always reflect the exact picture in real clinical practice. The actual benefit in terms of clinical outcome, viz., physician's satisfaction, cost-effectiveness, etc. must be proven by appropriate methods. AI-based models having either inaccuracies or those that deliver results divorced from clinical reality are likely to cause ethical issues owing to misdiagnosis or misclassification. Thus, the impact of $\mathrm{AI}$ application on the traditional doctor-patient relationship, which is the essence of the practice of medicine, should be looked into carefully. Ethical principles relevant to AI-based models (akin to Asimov's laws of robotics!) might need to be developed to tackle problems concerning medical ethics and AI. Finally, the formulation of reasonable regulatory guidelines and devising a proper reimbursement policy keeping in mind the economic aspects of health care are essential before integrating AI technology into the current health care structure. It is to be remembered that $\mathrm{AI}$ is not perfect and error-free. That is why the concept of "augmented intelligence" has emerged that emphasizes on improving and enhancing human intelligence rather than replacing it. The future challenges in the field of AI-based technology are described in $\boldsymbol{- T a b l e ~} 4$.

\section{Conclusion}

AI remains an ever-widening field with vast opportunities. In the domain of small bowel diseases, WCE seems to be the only modality at present where ML has been successfully applied in the form of lesion detection and localization. Although the scope of therapeutic intervention is limited, it is hoped that the use of appropriate AI-based technology cannot only shorten the examination time in WCE but also reduce intra/inter observer's variations and eliminate human bias. There is still a lot to be explored in the field of AI including rigorous validation of ML/ DL-based models. Therefore, in the present context, these cannot replace clinicians in making a diagnosis. Overfitting, spectrum bias, and lack of explainability remain the core concerns in the development of appropriate AI models. The ethical issues and the economic impact must be addressed judiciously as the day might not be far away when DL methods could be at the forefront among the scheme of modalities in diagnosis making. Real-time diagnosis using $\mathrm{AI}$ is a challenge and so also is the development of therapeutic options. To conclude, AI-based models, as of now, can only be used as an adjunct to the physician's clinical acumen and skills. However, the rapid pace at which $\mathrm{AI}$ is advancing promises exciting times in the field of endoscopic diagnosis and interventions in the GI tract, especially the small bowel.

\section{Author Contributions}

S.P.S. contributed toward conceptualization, methodology, data curation, writing, reviewing, and editing.

D.M., P.B., M.G., and P.A. contributed toward data curation and writing-original draft preparation.

All authors approved the final version of the article, including the authorship list.

Table 4 Future challenges in artificial intelligence

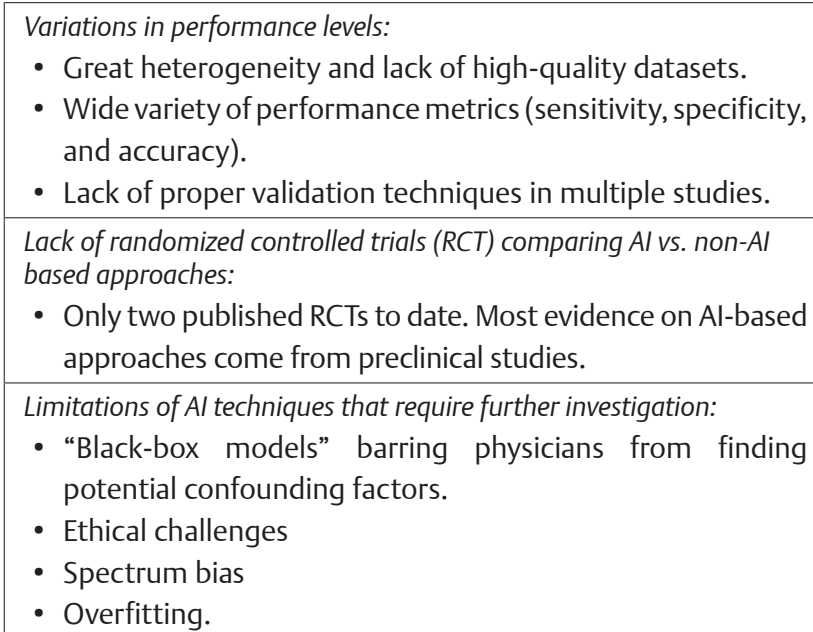

Abbreviation: Al, artificial intelligence. 


\section{Conflict of Interest}

None declared.

\section{Acknowledgments}

This work was partially supported by a grant from the Kalinga Gastroenterology Foundation, Cuttack, India.

\section{References}

1 Russell S, Norvig P, Artificial Intelligence: A Modern Approach. 4th ed. Upper Saddle River, NJ: Pearson Education, Inc; 2020

2 Mahmud M, Kaiser MS, Hussain A, Vassanelli S. Applications of deep learning and reinforcement learning to biological data. IEEE Trans Neural Netw Learn Syst 2018;29(6):2063-2079

3 Montavon G, Samek W, Müller K-R. Methods for interpreting and understanding deep neural networks. Digit Signal Process 2018;73:1-15

4 Ahmad OF, Soares AS, Mazomenos E, et al. Artificial intelligence and computer-aided diagnosis in colonoscopy: current evidence and future directions. Lancet Gastroenterol Hepatol 2019;4(1):71-80

5 Le Berre C, Sandborn WJ, Aridhi S, et al. Application of artificial intelligence to gastroenterology and hepatology. Gastroenterology 2020;158(1):76-94.e2

6 Iddan G, Meron G, Glukhovsky A, Swain P. Wireless capsule endoscopy. Nature 2000;405(6785):417

7 Han S, Fahed J, Cave DR. Suspected blood indicator to identify active gastrointestinal bleeding: a prospective validation. Gastroenterol Res 2018;11(2):106-111

8 Monteiro S, de Castro FD, Carvalho PB, Moreira MJ, Rosa B, Cotter J. PillCam ${ }^{\circledR}$ SB3 capsule: does the increased frame rate eliminate the risk of missing lesions? World J Gastroenterol 2016;22(10):3066-3068

9 Shiotani A, Honda K, Kawakami M, et al. Analysis of small-bowel capsule endoscopy reading by using Quickview mode: training assistants for reading may produce a high diagnostic yield and save time for physicians. J Clin Gastroenterol 2012;46(10):e92-e95

10 Xavier S, Monteiro S, Magalhães J, Rosa B, Moreira MJ, Cotter J. Capsule endoscopy with PillCamSB2 versus PillCamSB3: has the improvement in technology resulted in a step forward? Rev Esp Enferm Dig 2018;110(3):155-159

11 Constantinescu AF, Ionescu M, Iovănescu VF, et al. A computer-aided diagnostic system for intestinal polyps identified by wireless capsule endoscopy. Rom J Morphol Embryol 2016;57(3):979-984

12 Li B, Meng MQ-H, Lau JYW. Computer-aided small bowel tumor detection for capsule endoscopy. Artif Intell Med 2011;52(1):11-16

13 Liu G, Yan G, Kuang S, Wang Y. Detection of small bowel tumor based on multi-scale curvelet analysis and fractal technology in capsule endoscopy. Comput Biol Med 2016;70:131-138

14 Nawarathna R, Oh J, Muthukudage J. et al. Abnormal image detection in endoscopy videos using a filter bank and local binary patterns. Neurocomputing. 2014; 144: 70-91

15 Schulmann K, Hollerbach S, Kraus K, et al. Feasibility and diagnostic utility of video capsule endoscopy for the detection of small bowel polyps in patients with hereditary polyposis syndromes. Am J Gastroenterol 2005;100(1):27-37

16 Burke CA, Santisi J, Church J, Levinthal G. The utility of capsule endoscopy small bowel surveillance in patients with polyposis. Am J Gastroenterol 2005;100(7):1498-1502

17 Gupta A, Postgate AJ, Burling D, et al. A prospective study of MR enterography versus capsule endoscopy for the surveillance of adult patients with Peutz-Jeghers syndrome. AJR Am J Roentgenol 2010;195(1):108-116
18 Hakim FA, Alexander JA, Huprich JE, Grover M, Enders FT. CT-enterography may identify small bowel tumors not detected by capsule endoscopy: eight years experience at Mayo Clinic Rochester. Dig Dis Sci 2011;56(10):2914-2919

19 Ross A, Mehdizadeh S, Tokar J, et al. Double balloon enteroscopy detects small bowel mass lesions missed by capsule endoscopy. Dig Dis Sci 2008;53(8):2140-2143

20 Zhao Q, Dassopoulos T, Mullin GE, Meng MQ, Kumar R. A decision fusion strategy for polyp detection in capsule endoscopy. In J.D. Westwood, S.W. Westwood, L. Fellander-Tsai, R.S. Haluck, R.A. Robb, S. Senger, and K.G. Vosburgh, editors, Studies in health technology and informatics, volume 173 , pages 559 -565. IOS Press, 2012. Proceedings of the Medicine Meets Virtual Reality conference (MMVR19), Newport Beach, CA, USA, February 2012.

21 Li B, Meng M Q Xu L. A comparative study of shape features for polyp detection in wireless capsule endoscopy images. In Proceedings of the 2009 Annual International Conference of the IEEE Engineering in Medicine and Biology Society, Piscataway, NJ, USA, 3 -6 September 2009; pp. 3731 -3734.

22 Condessa F, Bioucas-Dias J. Segmentation and Detection of Colorectal Polyps Using Local Polynomial Approximation. In: Campilho A., Kamel M. (eds) Image Analysis and Recognition. ICIAR 2012. Lecture Notes in Computer Science, vol 7325. Springer, Berlin, Heidelberg.

23 Faghih Dinevari V, Karimian Khosroshahi G, Zolfy Lighvan M. Singular value decomposition based features for automatic tumor detection in wireless capsule endoscopy images. Appl Bionics Biomech. 2016; 2016: 3678913

24 Swain P. Wireless capsule endoscopy and Crohn's disease. Gut 2005;54(3):323-326

25 Sidhu R, McAlindon ME, Drew K, Hardcastle S, Cameron IC, Sanders DS. Evaluating the role of small-bowel endoscopy in clinical practice: the largest single-centre experience. Eur J Gastroenterol Hepatol 2012;24(5):513-519

26 Goldstein JL, Eisen GM, Lewis B, Gralnek IM, Zlotnick S, Fort JG; Investigators. Video capsule endoscopy to prospectively assess small bowel injury with celecoxib, naproxen plus omeprazole, and placebo. Clin Gastroenterol Hepatol 2005;3(2):133-141

27 Rosa B, Moreira MJ, Rebelo A, Cotter J. Lewis Score: a useful clinical tool for patients with suspected Crohn's Disease submitted to capsule endoscopy. J Crohn's Colitis 2012;6(6):692-697

28 Niv Y, Ilani S, Levi Z, et al. Validation of the capsule endoscopy Crohn's disease activity index (CECDAI or Niv score): a multicenter prospective study. Endoscopy 2012;44(1):21-26

29 Girgis HZ, Mitchell BR, Dassopouios T, Mullin G, Haga G. An intelligent system to detect Crohn's disease inflammation in wireless capsule endoscopy videos. Paper presented at: Proceedings of the 2010 IEEE International Conference on Biomedical Imaging: From Nano to Macro. ISBI'10. IEEE Press; 2010:1373-1376

30 Kumar R, Zhao Q Seshamani S, Mullin G, Hager G, Dassopoulos T. Assessment of Crohn's disease lesions in wireless capsule endoscopy images. IEEE Trans Biomed Eng 2012;59(2):355-362

31 Wei Z, Wang W, Bradfield J, et al; International IBD Genetics Consortium. Large sample size, wide variant spectrum, and advanced machine-learning technique boost risk prediction for inflammatory bowel disease. Am J Hum Genet 2013;92(6):1008-1012

32 Charisis VS, Hadjileontiadis LJ. Potential of hybrid adaptive filtering in inflammatory lesion detection from capsule endoscopy images. World J Gastroenterol 2016;22(39):8641-8657

33 Van de Bruaene C, De Looze D, Hindryckx P. Small bowel capsule endoscopy: where are we after almost 15 years of use? World J Gastrointest Endosc 2015;7(1):13-36 
34 Pais WP, Duerksen DR, Pettigrew NM, Bernstein CN. How many duodenal biopsy specimens are required to make a diagnosis of celiac disease? Gastrointest Endosc 2008;67(7):1082-1087

35 Ciaccio EJ, Tennyson CA, Bhagat G, Lewis SK, Green PH. Classification of videocapsule endoscopy image patterns: comparative analysis between patients with celiac disease and normal individuals. Biomed Eng Online 2010;9:44

36 Tenório JM, Hummel AD, Cohrs FM, Sdepanian VL, Pisa IT, de Fátima Marin H. Artificial intelligence techniques applied to the development of a decision-support system for diagnosing celiac disease. Int J Med Inform 2011;80(11):793-802

37 Gadermayr M, Kogler H, Karla M, Merhof D, Uhl A, Vécsei A. Computer-aided texture analysis combined with experts' knowledge: Improving endoscopic celiac disease diagnosis. World J Gastroenterol 2016;22(31):7124-7134

38 Zhou T, Han G, Li BN, et al. Quantitative analysis of patients with celiac disease by video capsule endoscopy: a deep learning method. Comput Biol Med 2017;85:1-6

39 Liedlgruber M, Uhl A. Computer-aided decision support systems for endoscopy in the gastrointestinal tract: a review. IEEE Rev Biomed Eng 2011;4:73-88

40 Charisis VS, Hadjileontiadis LJ, Liatsos CN, Mavrogiannis CC, Sergiadis GD. Capsule endoscopy image analysis using texture information from various colour models. Comput Methods Programs Biomed 2012;107(1):61-74

41 Chen Y, Lee J. Ulcer detection in wireless capsule endoscopy video. Paper presented at: Proceedings of the 20th ACM International Conference on Multimedia - MM '12. ACM Press; 2012:1181

42 Eid A, Charisis VS, Hadjileontiadis LJ, Sergiadis GD. A curvelet-based lacunarity approach for ulcer detection from Wireless Capsule Endoscopy images. Paper presented at: Proceedings of the 26th IEEE Int Symp Comput-Based Med Syst. Porto, Portugal. Piscataway (NJ); 2013

43 Yuan Y, Wang J, Li B, Meng MQ-H. Saliency based ulcer detection for wireless capsule endoscopy diagnosis. IEEE Trans Med Imaging 2015;34(10):2046-2057

44 Gupta R, Lakhtakia S, Tandan M, et al. Capsule endoscopic features of intestinal lymphangiectasia. Dig Endosc 2006;18(2):141-143

45 Cui L, Hu C, Zou Y, Song S, He Q, Meng MQ-H. Detection of lymphangiectasia disease from wireless capsule endoscopy images with adaptive threshold. Paper presented at: 2010 8th World Congr Intell Control Autom; Jinan, China 2010

46 Blanco-VelascoG, Solorzano-Pineda O, Hernández-Mondragón OV. Overt gastrointestinal bleeding caused by hookworm infection, diagnosed by capsule endoscopy. Dig Endosc 2018;30(4):531-532

47 He J-Y, Wu X, Jiang Y-G, Peng Q Jain R. Hookworm detection in wireless capsule endoscopy images with deep learning. IEEE Trans Image Process 2018;27(5):2379-2392

48 Wu X, Chen H, Gan T, Chen J, Ngo C-W, Peng Q. Automatic hookworm detection in wireless capsule endoscopy images. IEEE Trans Med Imaging 2016;35(7):1741-1752

49 Raju GS, Gerson L, Das A, Lewis B; American Gastroenterological Association. American Gastroenterological Association (AGA) Institute technical review on obscure gastrointestinal bleeding. Gastroenterology 2007;133(5):1697-1717

50 Robinson CA, Jackson C, Condon D, Gerson LB. Impact of inpatient status and gender on small-bowel capsule endoscopy findings. Gastrointest Endosc 2011;74(5):1061-1066

51 Lecleire S, Iwanicki-Caron I, Di-Fiore A, et al. Yield and impact of emergency capsule enteroscopy in severe obscure-overt gastrointestinal bleeding. Endoscopy 2012;44(4):337-342

52 Saperas E, Dot J, Videla S, et al. Capsule endoscopy versus computed tomographic or standard angiography for the diagnosis of obscure gastrointestinal bleeding. Am J Gastroenterol 2007;102(4):731-737

53 Milano A, Balatsinou C, Filippone A, et al. A prospective evaluation of iron deficiency anemia in the GI endoscopy setting: role of standard endoscopy, videocapsule endoscopy, and CT-enteroclysis. Gastrointest Endosc 2011;73(5):1002-1008

54 de Leusse A, Vahedi K, Edery J, et al. Capsule endoscopy or push enteroscopy for first-line exploration of obscure gastrointestinal bleeding? Gastroenterology 2007;132(3):855-862, quiz 1164-1165

55 Kameda N, Higuchi K, Shiba $M$, et al. A prospective, single-blind trial comparing wireless capsule endoscopy and double-balloon enteroscopy in patients with obscure gastrointestinal bleeding. J Gastroenterol 2008;43(6):434-440

56 Li B, Meng MQ-H. Computer-based detection of bleeding and ulcer in wireless capsule endoscopy images by chromaticity moments. Comput Biol Med 2009;39(2):141-147

57 Pan G, Yan G, Qiu X, Cui J. Bleeding detection in wireless capsule endoscopy based on probabilistic neural network. J Med Syst 2011;35(6):1477-1484

58 Lv G, Yan G, Wang Z. Bleeding detection in wireless capsule endoscopy images based on color invariants and spatial pyramids using support vector machines. Paper presented at: Conf Proc Annu Int Conf IEEE Eng Med Biol Soc IEEE Eng Med Biol Soc Annu Conf; Boston, MA, USA 2011:6643-6646

59 Fu Y, Zhang W, Mandal M, Meng MQ-H. Computer-aided bleeding detection in WCE video. IEEE J Biomed Health Inform 2014;18(2):636-642

60 Sainju S, Bui FM, Wahid KA. Automated bleeding detection in capsule endoscopy videos using statistical features and region growing. J Med Syst 2014;38(4):25

61 Hassan AR, Haque MA. Computer-aided gastrointestinal hemorrhage detection in wireless capsule endoscopy videos. Comput Methods Programs Biomed 2015;122(3):341-353

62 Schmit A, Gay F, Adler M. Cremer M, Van Gossum A. Diagnostic efficacy of push-enteroscopy and long-term follow-up of patients with small bowel angiodysplasias. Dig Dis Sci 1996;41(12):2348-2352

63 Leenhardt R, Vasseur P, Li C, et al; CAD-CAP Database Working Group. A neural network algorithm for detection of GI angiectasia during small-bowel capsule endoscopy. Gastrointest Endosc 2019;89(1):189-194

64 Tsuboi A, Oka S, Aoyama K, et al. Artificial intelligence using a convolutional neural network for automatic detection of small-bowel angioectasia in capsule endoscopy images. Dig Endosc 2020;32(3):382-390

65 England JR, Cheng PM. Artificial intelligence for medical image analysis: a guide for authors and reviewers. AJR Am J Roentgenol 2019;212(3):513-519

66 Park SH, Han K. Methodologic guide for evaluating clinical performance and effect of artificial intelligence technology for medical diagnosis and prediction. Radiology 2018;286(3):800-809

67 Park SH, Do K-H, Kim S, Park JH, Lim Y-S. What should medical students know about artificial intelligence in medicine? J Educ Eval Health Prof 2019;16:18

68 Bae H-J, Kim C-W, Kim N, et al. A perlin noise-based augmentation strategy for deep learning with small data samples of HRCT images. Sci Rep 2018;8(1):17687

69 Tavanaei A, Ghodrati M, Kheradpisheh SR, Masquelier T, Maida A. Deep learning in spiking neural networks. Neural Netw 2019;111:47-63 